\title{
A Preliminary Study on the Pathogenesis of Colorectal Cancer by Constructing a Hsa-circRNA- 0067835-miRNA-mRNA Regulatory Network
}

\author{
Ning Liu $\mathbb{D}^{1, *}$ \\ Fan Jiang ${ }^{2, *}$ \\ Zhiju Chen' \\ 'Department of Gastrointestinal Surgery, \\ Hainan General Hospital, Hainan \\ Affiliated Hospital of Hainan Medical \\ University, Haikou, 5703II, People's \\ Republic of China; ${ }^{2}$ Department of the \\ Center of Gerontology, Hainan General \\ Hospital, Hainan Affiliated Hospital of \\ Hainan Medical University, Haikou, \\ 5703 II, People's Republic of China
}

*These authors contributed equally to this work
Background: Increasing evidence shows that circular RNAs (circRNAs) play a key role in the development of colorectal cancer (CRC). An interesting candidate RNA in this context is hsa-circRNA-0067835 (circIFT80), but its network of actions is still unclear.

Methods: Big data mining technology was used to explore the downstream microRNAs (miRNA) and messenger RNAs (mRNA) of the circIFT80 network. A regulatory network, comprising circIFT80 and its corresponding miRNAs and mRNAs, was derived to preliminarily explore the potential mechanism of circIFT80 in CRC. Finally, the proposed regulatory network was experimentally verified at the cellular level.

Results: A total of 6 miRNAs were screened, of which hsa-miR-197-3p, hsa-miR-370-3p and hsa-miR-377-5p may be the most potential downstream miRNAs of hsa-circRNA -0067835 in CRC. A total of 74 up-regulated genes with opposite miRNA expression were selected for subsequent verification. Gene Ontology (GO) and Kyoto Encyclopedia of Genes and Genomes (KEGG) databases revealed that the target genes occurred more frequently in cancer-related pathways. In addition, protein-protein interaction (PPI) analysis of the target genes revealed a set of involved genes from which the hubTop 10 genes were selected for further analysis. Moreover, circRNA-miRNA-hubTop 10 mRNA networks were constructed. According to this analysis, circIFT80 simultaneously regulates hsa-miR-197-3p, hsa-miR $-370-3 p$, and hsa-miR-377-5p, among which hsa-miR-370-3p seems to be associated with further genes that may be relevant to CRC development. Therefore, the proposed circIFT80/ hsa-miR-370-3p/WNT7B, SLC1A5, RCBTB1 and COL6A6 signal axes were subjected to experimental verification. It could be shown that circIFT80 was up-regulated in CRC tissues. The circIFT80 was able to inhibit apoptosis and promote proliferation, migration and invasion. Moreover, circIFT80 inhibited the expression of hsa-miR-370-3p and promoted the expression of COL6A6, RCBTB1, SLC1A5 and WNT7B in CRC cell lines. Dual luciferase reporter assays further validated that circIFT80 is able to bind to hsa-miR-370$3 \mathrm{p}$ which in turn targets WNT7B.

Conclusion: The circIFT80 may play a role in carcinogenesis through the new circIFT80/ hsa-miR-370-3p/WNT7B signal axis. These findings may provide potential biomarkers and therapeutic targets for the treatment of CRC.

Keywords: circIFT80-miRNA-mRNA, colorectal cancer, hubTop 10 gene

\section{Introduction}

Colorectal cancer (CRC) is the third most common multiple tumor worldwide while at the same time being one of the most common malignant ones in the digestive system. ${ }^{1}$ According to the American Cancer Society, in 2018, 140,250 new cases of
Correspondence: Ning Liu Tel +86 I3876065304

Email drliuning@hainmc.edu.cn 
CRC were diagnosed in the United States, of which 75,610 were men and 64,640 were women; associated with this were 50,630 deaths of which 27,390 and 23,240 were males and females, respectively. The morbidity and mortality rate ranked third among all types of cancers. ${ }^{2}$ At present, the treatment of CRC is still based on surgery, supplemented by radiotherapy and chemotherapy. However, due to the fact that clinical symptoms are rarely detected in early stages, most of the patients get diagnosed in late stages of the disease. These patients have lost not only the opportunity of surgical cure but worse, chemotherapy is not as effective as necessary, leading to an overall poor prognosis. Therefore, the study of the underlying molecular mechanisms of CRC development and identifying effective targets for the treatment of CRC is of the highest interest.

With the development of high-throughput sequencing technologies, enabling large-scale sequencing of a wide range of cancer types, including $\mathrm{CRC}$, it has been shown that almost all cancers display a large number of abnormally expressed RNA molecules at the transcriptome level. ${ }^{3,4}$ A gene expression network system, comprising messenger RNA (mRNA), microRNA (miRNA), long noncoding RNA (lncRNA) and circular RNA (circRNA), plays an important role in the development of CRC through the complex interaction of its constituents. Analyzing the interaction of relevant mRNAs and noncoding RNA (ncRNA), and their changes in expression levels are therefore of prime importance for the elucidation of the molecular mechanisms leading to CRC formation. At the same time, this strategy may lead to a more reliable diagnosis by the identification of relevant prognostic markers and therefore to new potential therapeutic targets.

CircRNAs are a new type of endogenous ncRNA molecules with a closed loop structure. ${ }^{5}$ Compared to linear RNAs, circRNA has no 5'-end cap or 3'-polyadenylate tail structure, but instead forms a covalently closed loop structure that is resistant to exonuclease digestion, such as that of RNase R, leading to an extreme stability of this type of molecule. ${ }^{6}$ In 2011, Salmena et al proposed a competitive endogenous RNA (ceRNA) hypothesis in which ncRNAs can actively regulate the expression of mRNA through competitive binding to shared miRNAs. ${ }^{7}$ In line with these findings, recent studies have shown that circRNAs increase the expression of oncogenes or tumor suppressor genes through ceRNA mechanisms and thus likely participate in the development of tumors. Moreover, it was shown that hsa-circRNA-0067835 (circIFT80) gene knockout can enhance the radiosensitivity of CRC through the miR-296-5p/IGF1R axis. ${ }^{8}$ CircIFT80 also promotes the progression of CRC through the miR-1236-3p/ HOXB7 axis. ${ }^{9}$ In summary, it shows that circIFT80 plays an important role in the tumor progression of CRC. Inhibition of circIFT80 may be a potential treatment strategy for CRC. However, the downstream regulatory mechanism of circIFT80 in CRC still needs further investigation.

In this study, we identified and analyzed the circIFT80miRNA-mRNA regulatory network through bioinformatics analysis. The regulatory network of circIFT80 comprises hsa-miR-370-3p/WNT7B, SLC1A5, RCBTB1 and COL6A6. In addition, we could show that circIFT80 is highly expressed in CRC tissues and cells, and circIFT80 can inhibit apoptosis and promote proliferation, migration and invasion. In CRC cell lines, circIFT80 inhibits the expression of hsa-miR-370-3p and promotes the expression of COL6A6, RCBTB1, SLC1A5 and WNT7B. The experiments further revealed the binding effect of circIFT80, to hsa-miR-370-3p, and WNT7B, confirming that circIFT80 can be used as a sponge for hsa-miR-370-3p to regulate the expression of WNT7B. The findings of this work may provide new insights into the pathogenesis of CRC.

\section{Materials and Methods Sequencing Data Collection}

As the most authoritative cancer gene information database, The Cancer Genome Atlas (TCGA) (https://portal.gdc.can cer.gov/) database covers 33 cancer types, contains more than 30,000 tumor samples and 20,000 gene expression information. In this study, the gdc-client tool was used to download the RNA-seq and miRNA-seq data of CRC from the TCGA database. The RNA-seq data included 41 normal and 480 tumor samples, whereas the miRNA-seq data included 33 normal and 546 tumor samples.

\section{Analysis of Differentially Expressed miRNA and mRNA}

Differential expression analysis was performed using the DEM Limma package (linear models for microarray data) under $\mathrm{R}$ to analyze the expression differences between miRNAs and mRNAs, as well as to reveal differentially expressed miRNAs (DEMs) and finally, differentially expressed genes (DEGs) when comparing CRC tissues and normal tissues. The significance level was set to $\mathrm{p}<0.05$ and $|\log 2 \mathrm{FC}|>1$ to identify DEMs and DEGs. ${ }^{10}$ 


\section{Prediction of circRNA Binding miRNAs and Determination of miRNA}

Circular RNA Interactome (CircInteractome, https://circin teractome.nia.nih.gov/ $)^{11}$ which is based on the human circRNA sequence included in CircBase (http://www.cir cbase.org) was used for the prediction of circRNA binding to miRNAs. The downstream function prediction analysis of circRNA was emphasized, including the prediction of circRNA binding miRNA and possible binding of downstream proteins, which can be used for circRNA molecular retrieval, PCR primer design, RNA knockdown sequence design and more. In this study, the database was used to predict the miRNA binding to circIFT80. Cytoscape 3.7.2 software (https://cytoscape.org/) was used to visualize the prediction results. The circIFT80 targeting miRNA, the final miRNA of this study, was intersected with the DEMs obtained from the sequencing data.

\section{Prediction of miRNA Target Genes and Determination of $\mathrm{mRNA}$}

For the prediction of miRNA target genes and the determination of mRNAs, several software tools have been used: the DIANA-microT (http://microrna.gr/microT) software is a miRNA target gene prediction tool developed based on experimental and computational biology methods; miRanda (http://www.microrna.org/microrna/home. do) was the first miRNA target gene prediction software developed in May 2003, has a wide range of applications and is not restricted to a single species; PicTar (http:// pictar.mdc-berlin.de/) uses custom calculation rules to identify the target of miRNAs; TargetScan (http://www. targetscan.org/) predicts target genes by searching for conserved $8 \mathrm{mer}$ and $7 \mathrm{mer}$ sites that match each miRNA seed region. In this study, DIANA-microT, miRanda, PicTar and TargetScan were used to predict miRNA target genes, and the target genes predicted by each tool were taken as the intersection. ${ }^{12}$ The target gene corresponding to the miRNA intersects with DEGs obtained by sequencing, leading to the final target gene for research.

\section{Functional Enrichment Analysis of Target Genes Using Gene Ontology (GO) and Kyoto Encyclopedia of Genes and Genomes (KEGG)}

The GO database divides the function of genes into three parts: cell component (CC), molecular function (MF), and biological process (BP). By employing the GO database, we determined which of our target genes were mainly related to the CC, MF and BP levels. The KEGG database, in addition to the annotation of gene function, moreover delivers information about which genes will likely participate in all pathways of the human body. In our study, we used the $\mathrm{R}$ language ClusterProfiler package to analyze $\mathrm{GO}$ and KEGG functional enrichment of target genes. ${ }^{13}$

\section{Protein-Protein Interaction (PPI) Analysis of Target Genes and Hub Gene Screening} The String database (https://string-db) was used to conduct protein-protein interaction (PPI) analysis on the target genes. The data was then imported into Cytoscape 3.7.2 software to visualize the network diagram. The CytohHubba plug-in of Cytoscape 3.7.2 software was used to screen the top 10 genes in the network.

\section{Network Mapping of circRNA-miRNA- Gene and circRNA-miRNA-Hub Gene}

The data were imported into Cytoscape 3.7.2 software and the "Merge" function was used to construct a circRNAmiRNA-gene and a circRNA-miRNA-hub gene network map.

\section{Cell Lines and Human Tissues}

Normal human colonic epithelial cells HcoEpic and human CRC cell lines HT-29 and SW480 were purchased from the American Type Culture Collection (ATCC; Manassas, VA, USA). The HT-29 and SW480 cell lines were cultured in RPMI-1640 culture medium (Gibco; Thermo Fisher Scientific, Inc., Waltham, MA, USA) containing $100 \mathrm{U} / \mathrm{mL}$ penicillin (Gibco), $100 \mu \mathrm{g} / \mathrm{mL}$ streptomycin (Gibco) and 10\% fetal bovine serum (FBS; Gibco); human normal colonic epithelial cells were cultured in Dulbecco's modified eagle medium (DMEM) (Invitrogen; Thermo Fisher Scientific, Inc.) containing 10\% FBS. The cells were cultured under standard conditions in an incubator $\left(37^{\circ} \mathrm{C}, 5 \% \mathrm{CO}_{2}\right)$. Eighteen cases of CRC and corresponding paracancerous tissues were extracted from CRC patient tissues resected by gastrointestinal surgery at Hainan General Hospital. The clinical specimens were diagnosed by at least three pathologists. All patients signed the informed consent form and the procedures of this study were approved by the Clinical Ethics Management Committee of Hainan General Hospital (Med-Eth-Re [2020] 199). 


\section{Cell Transfection}

The cells were inoculated in 6 -well plates with $5.0 \times 10^{5}$ cells per well and incubated at $37^{\circ} \mathrm{C}$ and $5 \% \mathrm{CO}_{2}$ for 24 hours. Sequence transfection was carried out in strict accordance with the instructions of the lipofectamine ${ }^{\mathrm{TM}} 2000$ transfection kit (Invitrogen; Thermo Fisher Scientific, Inc.) for $48 \mathrm{~h}$.

\section{Apoptosis Detection by Flow Cytometry} Annexin V-FITC/PI apoptosis detection kit (Abcam, ab14085) was used to detect apoptosis. The treated cells were cultured for $48 \mathrm{~h}$. After this, the cells were collected, washed twice with PBS solution, centrifuged, resuspended in $200 \mu \mathrm{L}$ binding buffer, mixed gently with $10 \mu \mathrm{L}$ Annexin V-FITC and $5 \mu \mathrm{L} \mathrm{PI}$, and incubated at room temperature for $15 \mathrm{~min}$ protected from light. After this, $300 \mu \mathrm{L}$ binding buffer was added to the samples. Apoptosis was detected by flow cytometry (FACS Calibur; BD Biosciences) at $488 \mathrm{~nm}$. The experiment was repeated three times.

\section{Wound Healing Assay}

After a culture period of $24 \mathrm{~h}$, the cell density reached about $90 \%$. Then, the head of a sterilizing gun was scratched perpendicular to the horizontal line behind the ruler. The cells were washed twice with $1 \times \mathrm{PBS}$ and $2 \%$ serum containing medium was added to continue the culture. The culture was placed in the same observation position, and the migration of cells was observed by microscope (Nikon, New York, USA) at 0 and $48 \mathrm{~h}$.

\section{Cell Counting Kit-8 (CCK-8) Assay}

CCK-8 kit (Dojindo molecular technologies, Inc.) was used to identify the number of living cells. After 48 hours of transfection and culture, the cells of each group were collected and counted: $5 \times 10^{3}$ cells were inoculated in a 96-well plate and cultured in an incubator. $20 \mu \mathrm{L} \mathrm{CCK}-8$ solution was added to each well at $24 \mathrm{~h}, 48 \mathrm{~h}, 72 \mathrm{~h}$ and 96 $\mathrm{h}$. The culture was terminated after a $4 \mathrm{~h}$ incubation period and the optical density (OD) values of each well were was measured with an enzyme immunoassay analyser (Thermo Fisher Scientific, Shanghai, China).

\section{Transwell Assay}

The transwell assay was performed as described by $\mathrm{Li}^{14}{ }^{14} 48$ $\mathrm{h}$ after transfection, the cells were re-suspended in serumfree RPMI-1640 medium. $50 \mu \mathrm{L}$ Matrigel (Becton-
Dickinson, CA, USA) was added to the upper chamber and air-dried at room temperature for $4 \mathrm{~h}$. The cell suspension with a cell density of $3 \times 10^{5} / \mathrm{mL}$ was added to the upper chamber of a 24-well transwell chamber. The lower transwell chamber was supplemented with $500 \mu \mathrm{L}$ RPMI-1640 medium containing $10 \%$ FBS per well, placed in an incubator for $24 \mathrm{~h}$, afterwards washed twice with PBS, and treated with methanol for $10 \mathrm{~min}$, followed by a WrightGiemsa staining. The fibrous membrane of the insert was removed and sealed. Six random visual fields of cells in each group were photographed and counted under the inverted phase-contrast microscope (Olympus Corporation).

\section{Dual Luciferase Reporter Assay}

The reporter vector constructs used comprised the following vectors: pmirGLO-negative control (NC), pmirGLOWNT7B-wild-type (wt), pmirGLO-WNT7B-mutant (mut), pmirGLO-circIFT80-wt and pmirGLO-circIFT80-mut plasmids, synthetic miR-NC mimics or hsa-miR-370-3p mimics (Sangon Biotech Co., Ltd. Shanghai, China). For transfection, $70-80 \%$ confluent $293 \mathrm{~T}$ cells were cotransfected with reporter plasmids miR-NC mimics or hsamiR-370-3p mimics using Lipofectamine ${ }^{\circledR} 2000$ (Invitrogen; Thermo Fisher Scientific, Inc.). $24 \mathrm{~h}$ after transfection, the relative firefly luciferase activity was detected using a Dual-Glo Luciferase Reporter assay system (Promega, USA).

\section{Western Blotting}

Total protein was extracted from the cells using RIPA lysis buffer (Beyotime Ltd., Beijing, China), quantified using a BCA protein assay kit (Bio-Rad Laboratories, Hercules, USA) and separated via a $12 \%$ SDS-PAGE (NuPAGE; Invitrogen; Thermo Fisher Scientific, Inc.). The separated proteins were subsequently transferred onto a PVDF membrane (Whatman plc; GE Healthcare Life Sciences) and blocked with 5\% non-fat dry milk powder (Merck KGaA) at room temperature for $2 \mathrm{~h}$. The PVDF membrane was then incubated with RCBTB1 (1:1000, Immunoway, YT0974), SLC1A5 (1:1000, Immunoway, YT7482), WNT7B (1:1000, Immunoway, YN0288) and Tubulin (1:5000, Abcam, ab7291) primary antibodies at $4^{\circ} \mathrm{C}$ overnight. Following the primary antibody incubation, the membranes were washed with TBS-Tween 20 three times and incubated with a goat anti-rabbit secondary antibody (1:3000, Bioss, Beijing China) for $2 \mathrm{~h}$ at room temperature. Protein bands were visualized using a chemiluminescence reagent on a gel imaging system. The gray value of the target protein was 
normalized to the gray value of the internal reference protein. Tubulin and the data are presented as the relative content of the target protein in a sample.

\section{RNA Extraction and Quantitative RT-PCR (qRT-PCR)}

Total RNA was extracted from cells and human tissues by TRIzol reagent (Invitrogen; Thermo Fisher Scientific, Inc). According to the PrimeScript RT reagent kit (Takara, Japan) instructions, the total RNA was reverse transcribed into cDNA. The synthesized cDNA was detected by qRTPCR with the Fast SYBR Green PCR kit (Biosharp, Inc) and an ABI PRISM 7300 RT-PCR system (Model: ABI7300, Applied Biosystems; Thermo Fisher Scientific, Inc.). U6 and GAPDH were used as internal references. The relative expression of each gene was calculated by the relative quantitation method $\left(2^{-\Delta \Delta C \mathrm{~T}}\right)$. The experiment was repeated three times. Primer 5 web software (version 4.0.0) was used to design the following primers for the qPCR. The primer sequences are shown in Table 1.

\section{Statistical Analysis}

The data were analyzed using SPSS 17.0 statistical software (IBM Corporation). Three independent experiments were performed, and the data are presented as the mean \pm standard deviation. The unpaired Student's $t$-test was applied for comparing two groups, and the one-way analysis of variance (ANOVA) was applied for comparing multiple groups. ${ }^{* *} \mathrm{p}<0.01,{ }^{*} \mathrm{p}<0.05$ indicates a significant difference.

\section{Results}

\section{Screening of DEMs and DEGs in CRC}

The sequencing data of CRC miRNA and mRNA were downloaded from TCGA database and the screening criteria were set at $|\log 2 \mathrm{FC}|>1$ and $\mathrm{p}<0.05 .402$ DEMs were obtained of which 135 were up-regulated and 267 were down regulated (Figure 1A and B). 8738 DEGs were obtained of which 5430 were up-regulated and 3308 were down regulated (Figure 1C and D).

\section{Prediction of Hsa-circRNA-0067835 Binding miRNA}

The hsa-circRNA-0067835 binding miRNAs were chosen according to predictions calculated by the Circinteractome database. A total of 34 target miRNAs were obtained.

The targeting relationship can be seen in Figure 2A. The intersection of the hsa-circRNA-0067835 binding miRNA, the DEMs of the sequencing data and a total of 6 DEMs were obtained. Of these, 3 were up-regulated (hsa-miR-136-5p, hsamiR-186-5p, hsa-miR-577) and 3 down-regulated (hsa-miR -197-3p, hsa-miR-370-3p, hsa-miR-377-5p) (Figure 2B and C). We used the StarBase database to verify the expression levels of 6 DEMs. The results showed that the miRNA expression levels were consistent with previous analysis results (Figure 2D-I). Since circRNA is negatively correlated with miRNA, an upregulation of hsa-circRNA-0067835 in CRC should result in a down-regulation of its targeted miRNA. Therefore, hsa-circRNA-0067835/ hsa-miR-197-3p, hsa-miR -370-3p, and hsa-miR-377-5p signal axes were determined.

\section{Identification of miRNA Downstream Target Genes and Target Gene Enrichment Analysis}

For the prediction of the target genes of hsa-miR-197-3p, hsamiR-370-3p, and hsa-miR-377-5p, we employed the DIANA, miRanda, PicTar and TargetScan databases. A total of 713 overlapping results were obtained. Of these, 156 genes were obtained after intersecting the differential mRNAs screened with the mRNA sequencing data. Among them, 74 were upregulated and 82 were down-regulated genes (Figure $3 \mathrm{~A}$ and C) Since miRNA and mRNA are inversely correlated, the 74 up-regulated genes with the opposite expression of miRNA

Table I Primer Sequences

\begin{tabular}{|l|c|c|}
\hline Gene & Forward $\mathbf{( 5}^{\prime} \rightarrow \mathbf{3}^{\prime} \mathbf{)}$ & Reverse $\mathbf{( 5}^{\prime} \rightarrow \mathbf{3}^{\prime} \mathbf{)}$ \\
\hline circlFT80 & CCGCCCACTGTACAATTCAC \\
miR-370-5p & CAGGUCACGUCUCUGCAGUUAC & TCTTCAGCAGTAGTCCAGCC \\
COL6A6 & ACCCAACCGAGTTGGTGTTT & CGCATTGCTCTGCCAATCTC \\
RCBTBI & CCTCCTCTTCGCTGCCG & CCACTTTCCGACATCCACCA \\
SLCIA5 & CTCCAGCCCTCGGGAGTAAA \\
WNT7B & GACAAGTGCGGACACATTGG \\
U6 & CTCGCTTCGGCAGCACA & CTGTGCATGATCCAGGGAGG \\
GAPDH & GAAAGCCTGCCGGTGACTA & AACGCTTCACGAATTTGCGT \\
\hline
\end{tabular}


A

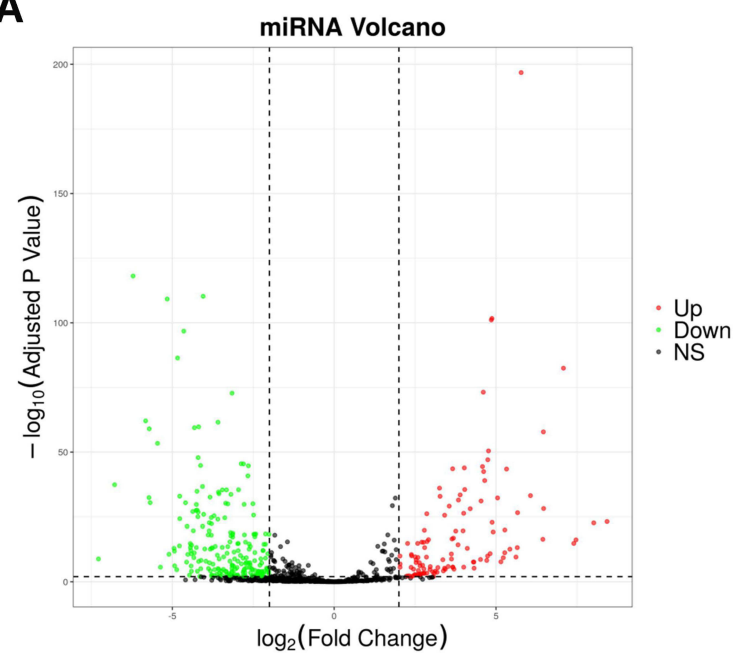

C

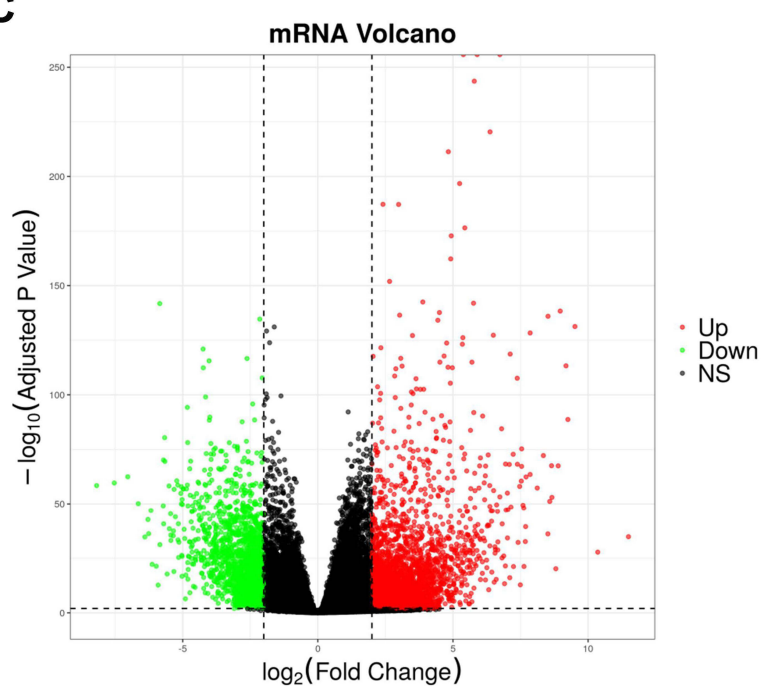

B

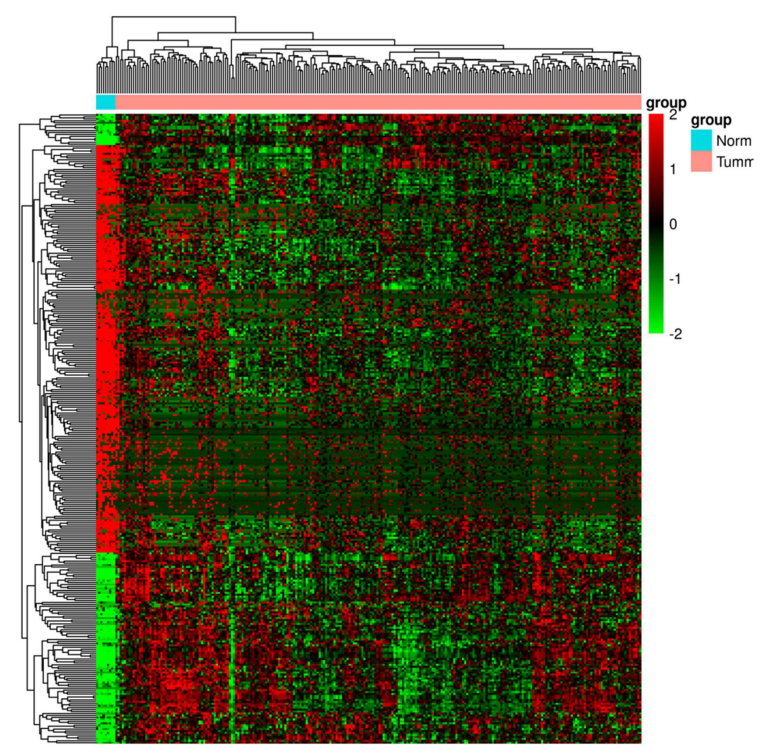

D

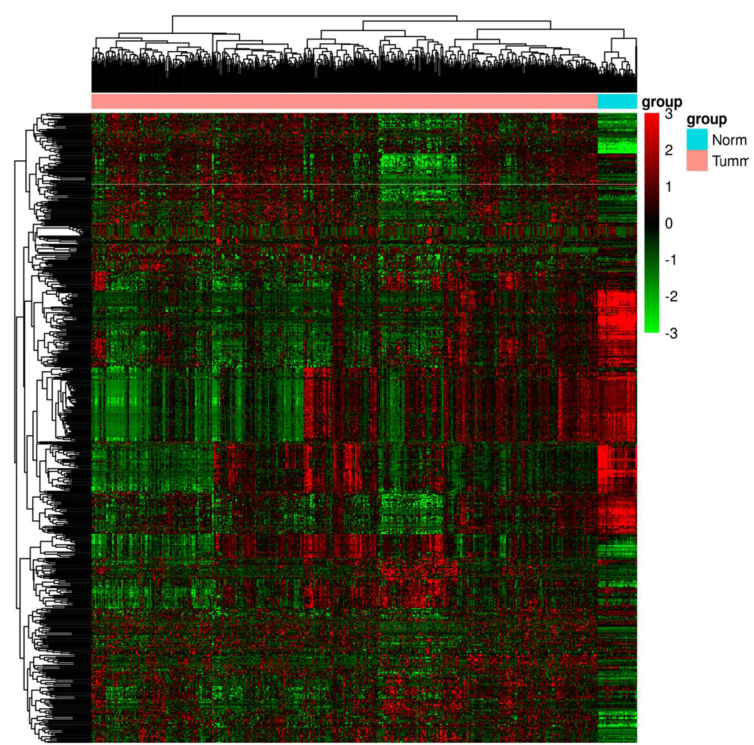

Figure I Identification of candidate DEMs and DEGs associated with progression of CRC; (A) The volcano plot of DEMs between tumor and normal tissue; (B) The heat map of DEMs; (C) The volcano plot of DEGs between tumor and normal tissue; (D) The heat map of DEGs.

were selected for subsequent verification. R language cluster profiler was used to analyze the GO and KEGG enrichment of these genes. GO analysis showed that these genes were not enriched in $\mathrm{CC}$; however, $\mathrm{BP}$ was mainly enriched in Eye development, visual system development and sensory system development; MF was mainly enriched in ligand-gated ion channel activity, ligand-gated channel activity and extracellular matrix structural constituent conferring tensile strength (Figure 3B); KEGG analysis showed that these genes were mainly enriched in signal pathways such as protein digestion and absorption, primary bile acid biosynthesis, and ECMreceptor interaction (Figure 3D).

\section{Hub Gene Screening and Enrichment Analysis}

Using a combination of String+Cytoscape results to visualize the target gene PPI network diagram (Figure 4A), the cytohHubba plug-in revealed the Top 10 hub genes, namely ITGB8, COL5A1, COL4A1, COL6A6, WNT7B, MYC, SLC1A5, TEAD4, RCBTB1 and CDCA2 (Figure 4B). Next, $\mathrm{R}$ language cluster profiler was used for $\mathrm{GO}$ and $\mathrm{KEGG}$ enrichment analysis of the Top 10 hub genes. The GO analysis showed that the genes were not enriched in $\mathrm{CC}$ and MF. However, BP was mainly enriched in renal tubule development, connective tissue development, and urogenital system 

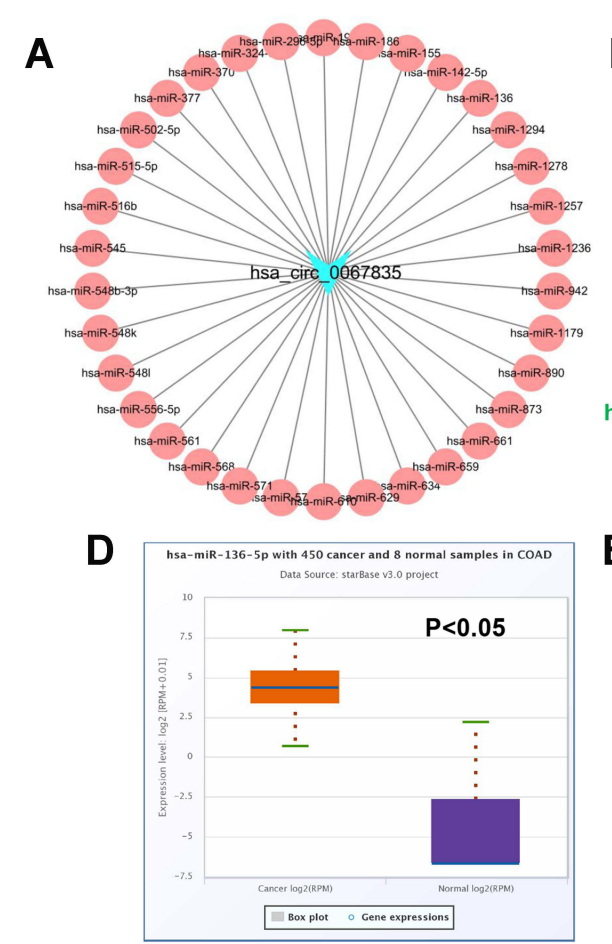

G

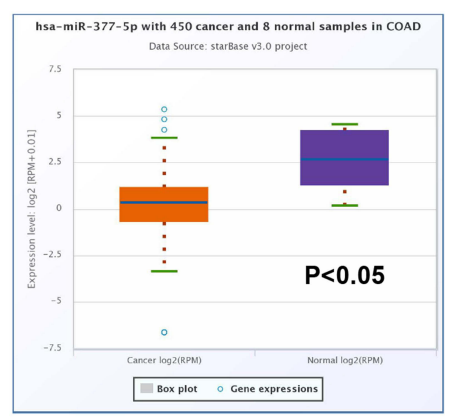

B

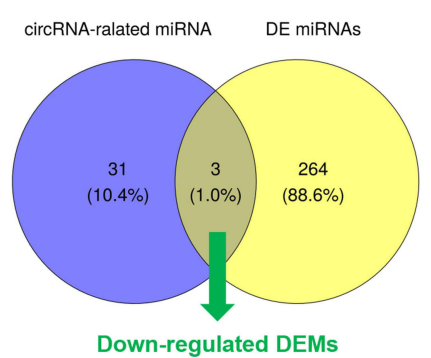

hsa-miR-197-3p, hsa-miR-370-3p, hsa-miR-377-5p
C

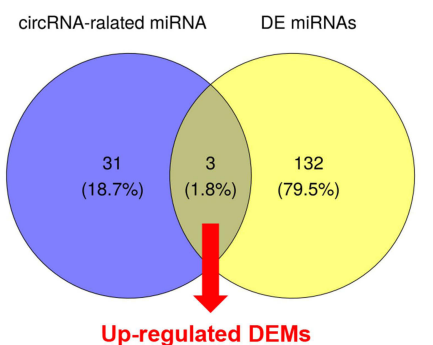

hsa-miR-136-5p, hsa-miR-186-5p, hsa-miR-577
E

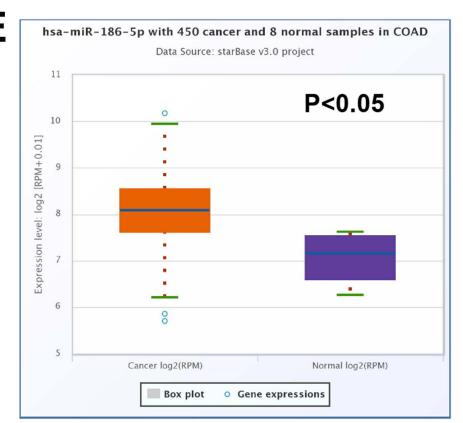

$\mathbf{H}$

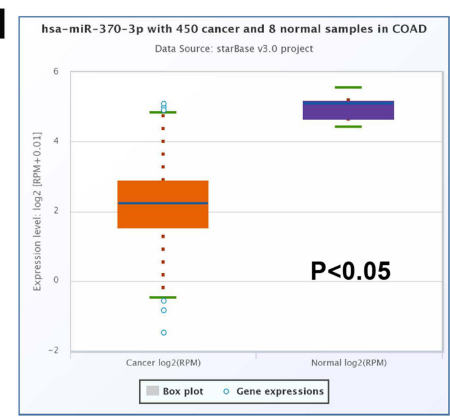

$\mathbf{F}$

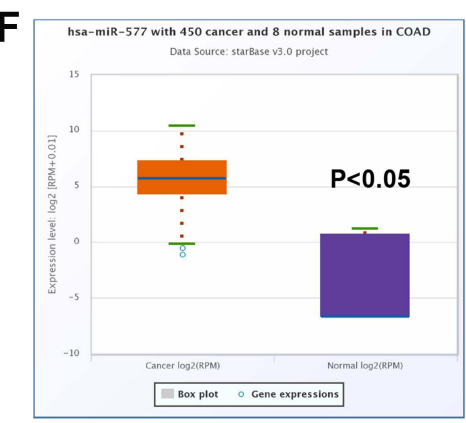

I

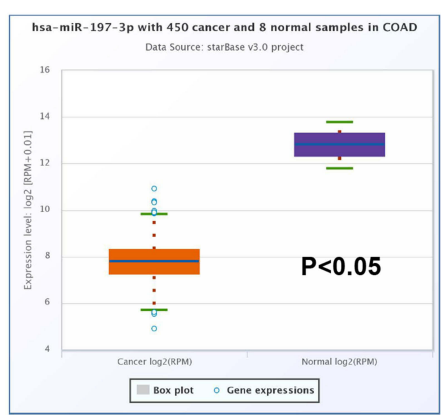

Figure 2 Hsa-circRNA-0067835-miRNA network diagram and identification of potential miRNAs bound by hsa-circRNA-0067835 in CRC. (A) Hsa-circRNA-0067835miRNA network diagram; (B) Down-regulated miRNA Venn diagram; (C) Up-regulated miRNA Venn diagram; (D) Expression level of hsa-miR-I36-5p; (E) Expression level of hsa-miR-I86-5p; (F) Expression level of hsa-miR-577; (G) Expression level of hsa-miR-197-3p; (H) Expression level of hsa-miR-370-3p; (I) Expression level of hsa-miR $-377-5$ p. $p<0.05$, CRC vs control.

development (Figure 4C). The KEGG analysis revealed that the genes were mainly enriched in signaling pathways related to cancer such as protein digestion and absorption, ECMreceptor interaction, human papillomavirus infection, the PI3K-Akt signaling pathway and Hippo signaling pathway (Figure 4D).

\section{CircRNA-miRNA-mRNA Network Diagram Construction}

We have constructed the circRNA-miRNA-mRNA and the circRNA-miRNA-Top10 hubgene network diagram. From the target gene network diagram, it is obvious that hsa-miR-370-3p connect to more genes, which may be a more important miRNA downstream of hsa-circRNA -0067835 (Figure 5A). The Top10 hubgene network diagram of circRNA-miRNA-hubTOP10 shows that hsacircRNA-0067835 simultaneously regulates hsa-miR -197-3p, hsa-miR-370-3p, hsa-miR-377-5p, and that its downstream hsa-miR-370-3p can simultaneously regulate WNT7B, SLC1A5, RCBTB1 and COL6A6 genes (Figure 5B). Therefore, we decided to select the hsacircRNA-0067835/hsa-miR-370-3p/WNT7B, SLC1A5, RCBTB1 and COL6A6 signal axes for experimental verification.

The Expression Levels of circlFT80 and Its Effect on Proliferation, Apoptosis, Migration, and Invasion of CRC Cell Lines QRT-PCR analysis not only detected the expression of circIFT80 in normal and cancer tissues as well as in cell 


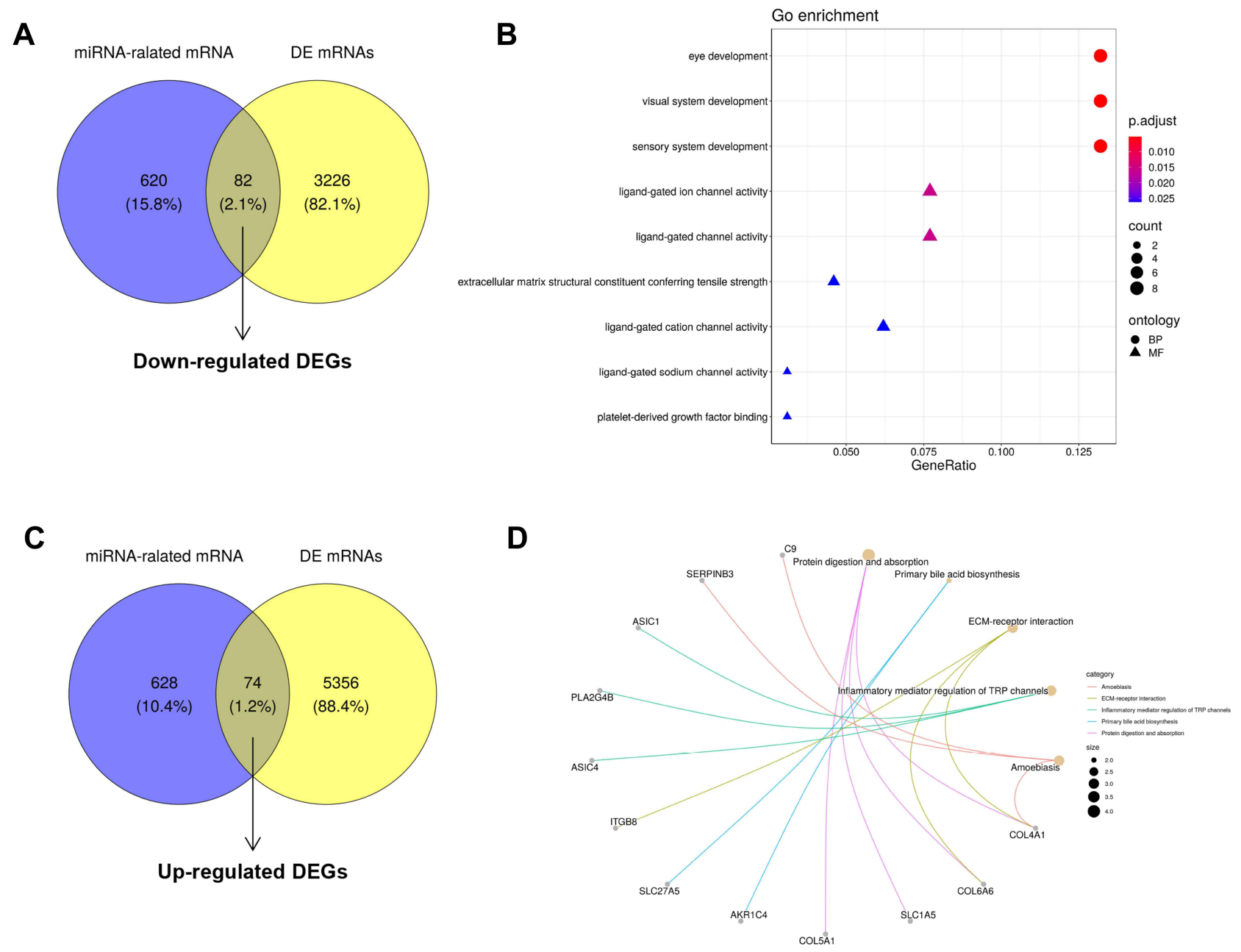

Figure 3 Identification of potential target genes that bind to hsa-miR-197-3p, hsa-miR-370-3p, and hsa-miR-377-5p in CRC and GO and KEGG enrichment analysis of target genes. (A) Down-regulated mRNA Venn diagram; (B) Target gene GO enrichment analysis; (C) Up-regulated mRNA Venn diagram; (D) Target gene KEGG enrichment analysis.

lines but also showed that circIFT80 was significantly upregulated (Figure 6A and B). Cell function experiments further verified the effect of circIFT80 on SW480 cells. In our study, we overexpressed and knockdown with circIFT80. The results of the wound healing assay showed that the overexpression of circIFT80 significantly increased the healing rate of SW480 cells, and the knockdown of circIFT80 significantly reduced the healing rate of the cells (Figure 6C and D). Transwell experiment results showed that overexpression of circIFT80 significantly promoted the invasiveness of SW480 cells, whereas the knockdown of circIFT80 inhibited invasiveness (Figure 6E and F). The CCK-8 assay results showed that the overexpression of circIFT 80 significantly promoted the proliferation of SW480 cells and the knockdown of circIFT80 inhibited SW480 cell proliferation (Figure 6G). The results of the apoptosis experiments showed that the overexpression of circIFT80 decreased and the knockdown of circIFT80 increased the apoptosis rate of these cells, respectively (Figure $6 \mathrm{H}$ and I). The above experimental results show that circIFT80 was able to inhibit apoptosis and promote SW480 cell proliferation, migration, and invasion.

\section{The Effect of circlFT80 on the Expression Levels of circlFT80, Hsa-miR-370-3p, COL6A6, RCBTBI, SLCIA5 and WNT7B in SW480 Cells and circlFT80 Upregulates WNT7B Expression via Sponging Hsa-miR-370-3p}

QRT-PCR results showed that after overexpression of circIFT80 in SW480 cells, circIFT80, COL6A6, RCBTB1, SLC1A5, WNT7B mRNA were significantly up-regulated 
A

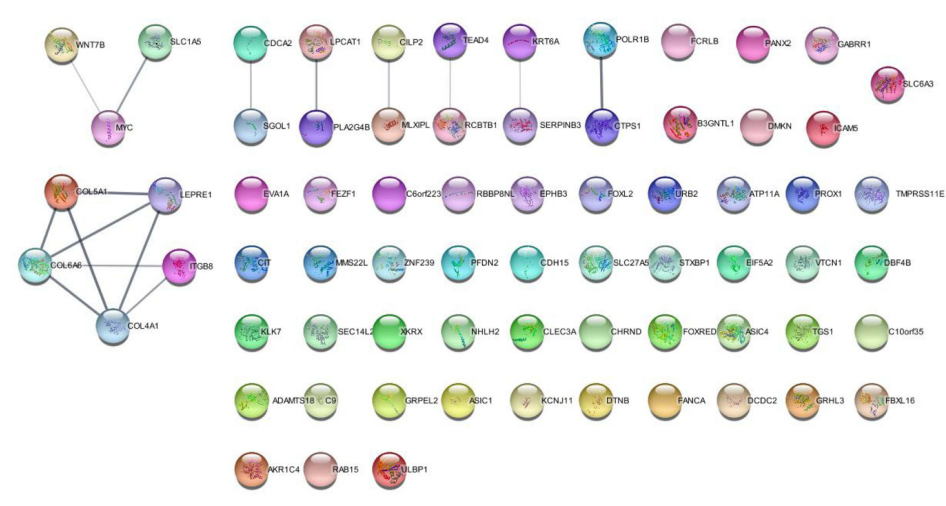

B
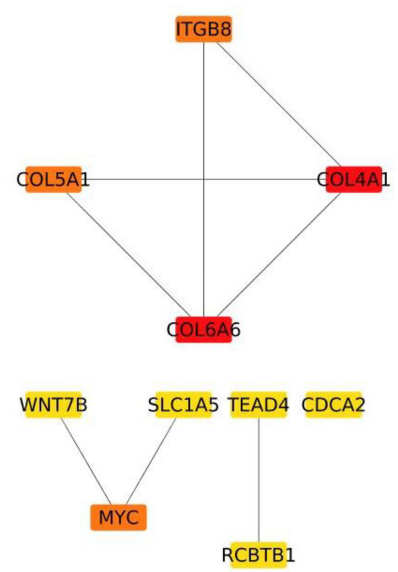
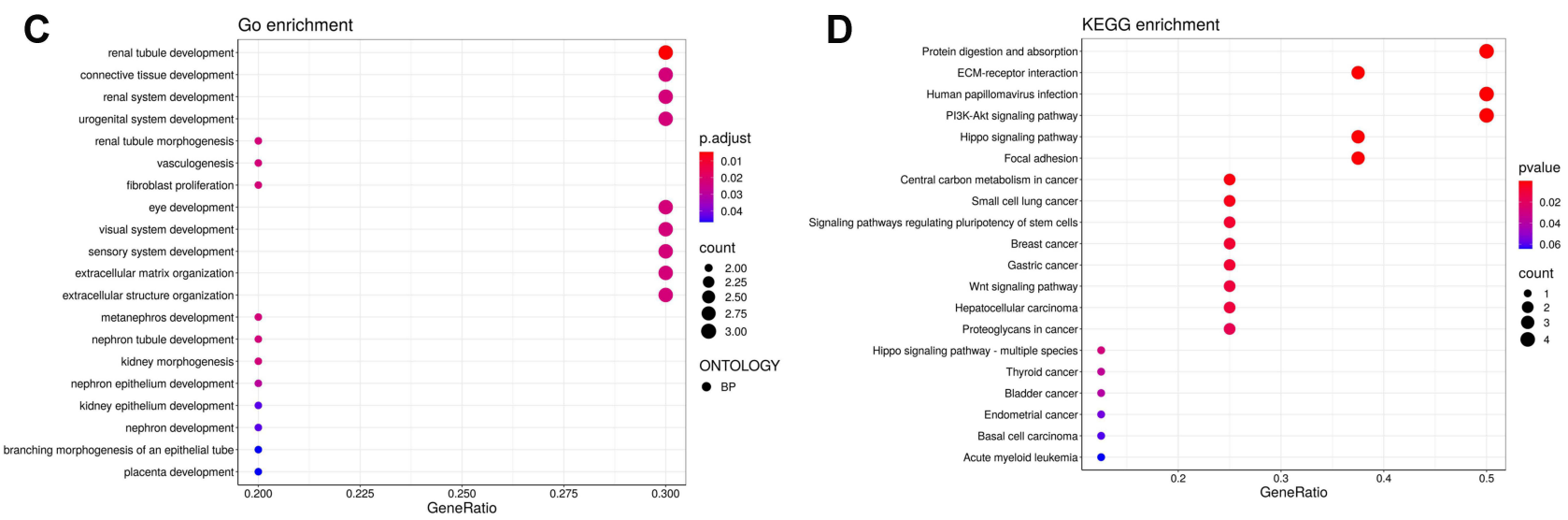

Figure 4 Top 10 hub gene screening and gene functional enrichment analysis. (A) Target gene PPI analysis results; (B) Top 10 hub gene screening results; (C) Top I0 hub gene GO enrichment analysis; (D) Top I0 hub gene KEGG enrichment analysis.

and hsa-miR-370-3p was significantly down-regulated. Moreover, after knockdown with circIFT80, hsa-miR-370$3 p$ was significantly up-regulated, as well as circIFT80, COL6A6, RCBTB1, SLC1A5, WNT7B mRNA was significantly down-regulated (Figure 7A-F). Western blot results showed that overexpression of circIFT80 increased the protein expression levels of RCBTB1, SLC1A5, and WNT7B; knockdown of circIFT80 inhibits the protein expression levels of RCBTB1, SLC1A5, and WNT7B (Figure 7G and H). Experimental results showed that circIFT80 was negatively correlated with hsa-miR-370-3p but was positively correlated with target genes COL6A6, RCBTB1, SLC1A5, and WNT7B. In order to further verify the regulatory effect of circIFT80 on its downstream miRNA and mRNA, we selected the circIFT80/hsa-miR-370-3p/WNT7B axis that we are more interested in for verification experiments. Bioinformatics analysis identified 14 complementary base pairings between hsa-miR-370-3p and circIFT80 (Figure 7I). Similarly, complementary base pairings were also identified between hsa-miR-370-3p and WNT7B
(Figure 7J). Dual luciferase reporter assay results revealed that circIFT80 can target hsa-miR-370-3p, and hsa-miR-370$3 \mathrm{p}$ can in turn target WNT7B. These findings suggested that circIFT80 may reduce the degradation of WNT7B mRNA by sponging hsa-miR-370-3p, thereby exerting a regulatory role over WNT7B (Figure 7K and L).

\section{Discussion}

An increasing number of studies show that circRNA plays a vital role in the occurrence and development of CRC. HsacircRNA-103809 regulates the proliferation and migration of CRC cells through the miR-532-3p/FOXO4 axis. $^{15}$ CircRNA-100290 down-regulates FZD4 expression and $\mathrm{Wnt} / \beta$-catenin signaling pathway through miR-516b resulting in a promotion of CRC progression. ${ }^{16}$ CircMBOAT2 is a new tumor marker that regulates the proliferation/migration of CRC by regulating miR-519d-3p. ${ }^{17}$ Although some studies have reported the construction of circRNA-miRNAmRNA regulatory network in $\mathrm{CRC},{ }^{18}$ the understanding of circRNA-related ceRNA regulatory networks in CRC is not 

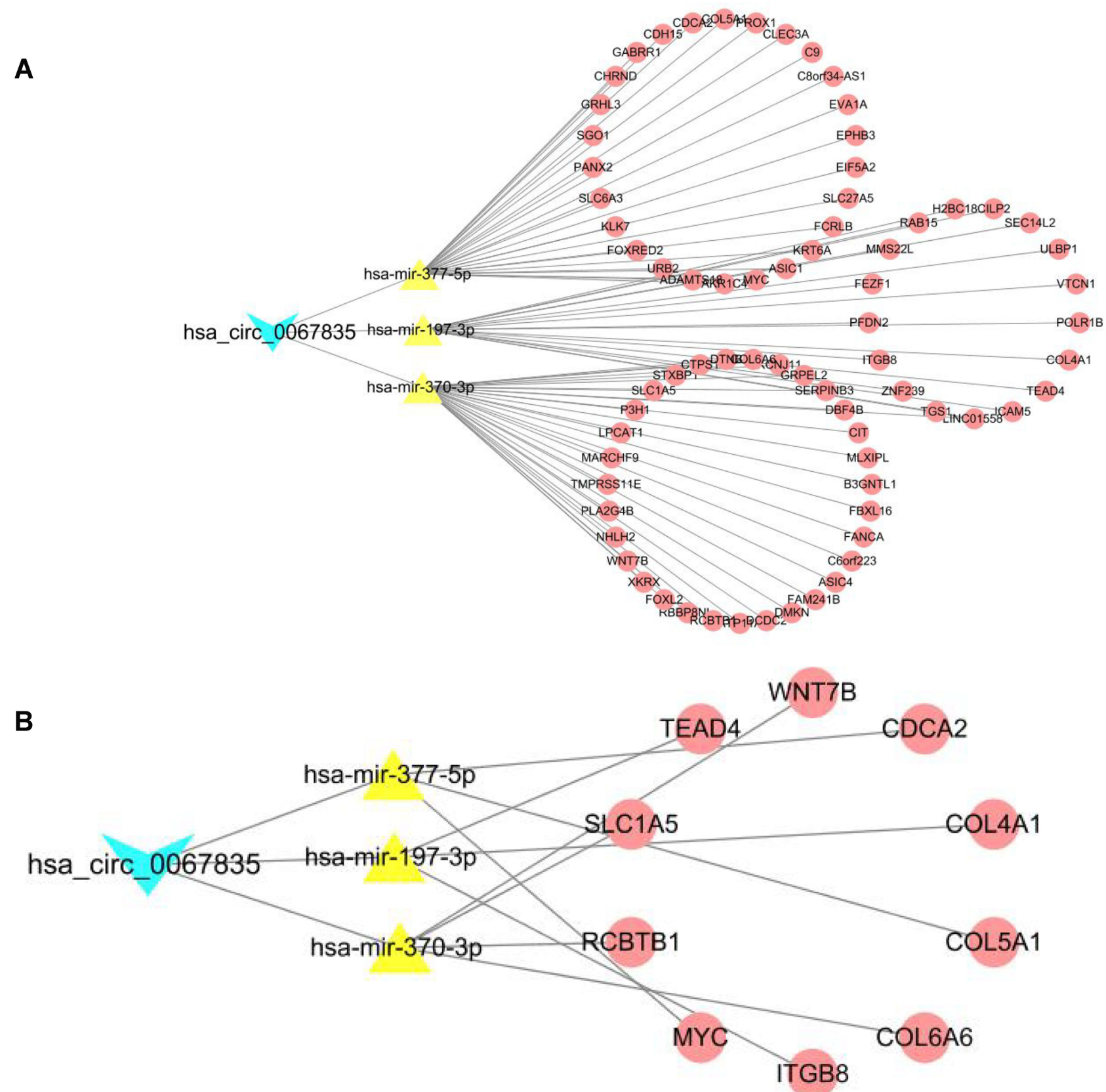

Figure 5 CircRNA-miRNA-mRNA network diagram. (A) CircRNA-miRNA-mRNA network diagram; (B) CircRNA-miRNA-TOPI0 hub gene network diagram.

sufficient and further research is needed to reveal the expression of circRNA and its mechanisms.

In this study, we focused on circIFT80 and explored its downstream targets through bioinformatics analysis. CircRNA has been proven to act as a miRNA sponge that is able to regulate gene expression. ${ }^{19,20}$ Therefore, we predicted the potential miRNAs of circIFT80 and were able to show that hsa-miR-197-3p, hsa-miR-370-3p, and hsa-miR -377-5p may be the most potential downstream miRNAs in CRC. In addition, the hsa-circRNA-0067835/hsa-miR-197- 3p, hsa-miR-370-3p, and hsa-miR-377-5p signal axes were initially determined. One study has shown that hsa-miR -370-3p reduces ulcerative colitis-related CRC by inhibiting inflammation and epithelial-mesenchymal transition; ${ }^{21}$ Hsa-circ-0002024 inhibits bladder cancer cell proliferation, migration and invasion by sponging miR-197-3p. ${ }^{22}$ The above results suggest that both hsa-miR-197-3p and hsamiR-370-3p are related to cancer. Next, we predicted the target genes of hsa-miR-197-3p, hsa-miR-370-3p, and hsamiR-377-5p and determined the target genes by GO and 


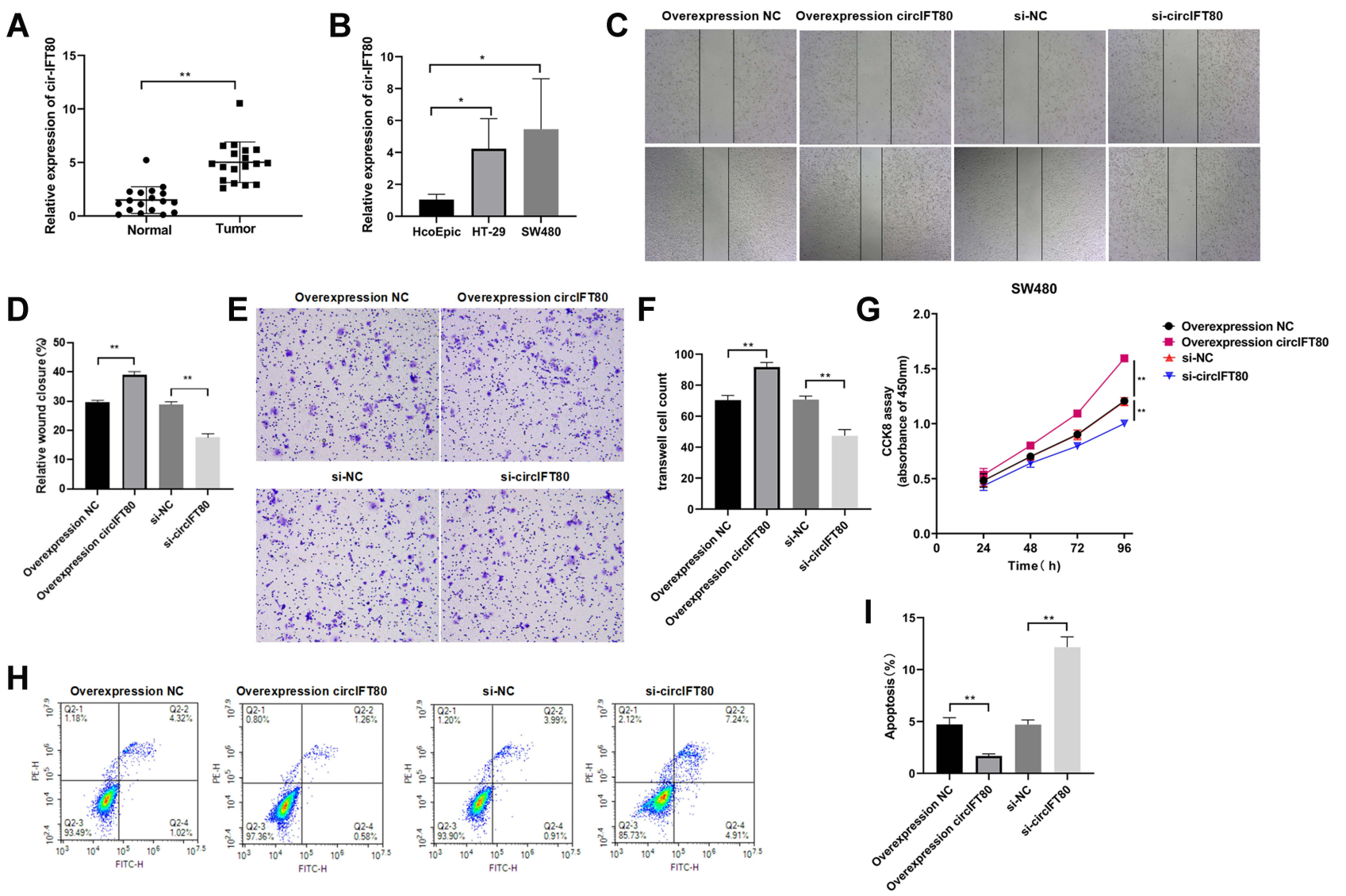

Figure 6 CirclFT80 is up-regulated in CRC tissues and cell lines (HcoEpic, HT-29, SW480) and circlFT80 is able to inhibit cell apoptosis and promote cell proliferation, migration, and invasion. (A) qRT-PCR assay was applied to detect the relative expression of circlFT80 in CRC tissues and adjacent tissues; (B) qRT-PCR assay was applied to detect the relative expression of circlFT80 in HcoEpic, HT-29, SW480 cell lines; (C and D) Wound-healing assay were used to detect the migration of SW480 cells after overexpression and knockdown with circlFT80; (E and F) Transwell assay was applied to detect the invasion of SW480 cells after overexpression and knockdown with circlFT80; (G) CCK8 assay were used to detect the viability of SW480 cells after overexpression and knockdown with circlFT80; (H) Flow cytometry were used to detect the apoptosis of SW480 cells after overexpression and knockdown with circlFT80; (I) Apoptosis rate. **p<0.01. ${ }^{*} p<0.05$. vs NC.

KEGG enrichment analysis. The results showed that they were enriched in cancer-related pathways. Then, PPI analysis was performed on the target genes. For this, the genes of Top10 hub (ITGB8, COL5A1, COL4A1, COL6A6, WNT7B, MYC, SLC1A5, TEAD4, RCBTB1 and CDCA2) were selected and the circRNA-miRNA-mRNA and circRNA-miRNA-hub Top 10mRNA networks were constructed. From the network diagram, it became obvious that circIFT80 regulates hsa-miR-197-3p, hsa-miR-370-3p, and hsa-miR-377-5p at the same time. Among them, hsamiR-370-3p connects to more target genes, indicating that it is more important in CRC, and hsa-miR-370-3p can regulate downstream WNT7B, SLC1A5, RCBTB1 and COL6A6 genes. Therefore, we decided to focus on the circIFT80/hsa-miR-370-3p/WNT7B， SLC1A5， RCBTB1 and COL6A6 signal axis for preliminary experimental verification. Previous studies have shown that miR-370-3p can increase the expression of E-cadherin and decrease the expression of $\mathrm{N}$-cadherin and Vimentin by regulating the expression of TLR4 and $\beta$-catenin in vivo, thereby significantly inhibiting inflammation and EMT, and by this, reducing Ulcerative Colitis-Related CRC. ${ }^{21}$ Moreover, it has been shown that WNT7B autocrine activation can reduce the metastasis of CRC epithelial to mesenchymal transition and at the same time predict a poor prognosis. ${ }^{23}$ SLC1A5 is up-regulated in CRC tissues and promotes the growth and survival of CRC cells. ${ }^{24}$ The low expression of RCBTB1 is a prognostic factor for the progression of leiomyosarcoma metastasis. In vitro and in vivo, inhibiting RCBTB1 can reduce proliferation and protect sarcoma cells from docetaxel-induced apoptosis. ${ }^{25}$ COL6A6 is down-regulated in pituitary adenoma tissues and cell lines which is closely related to poor survival. COL6A6, in addition, inhibits the growth and invasion of pituitary adenoma cells by inhibiting the PI3K-Akt pathway in vitro and in vivo. ${ }^{26}$ All the above studies suggest that the hsa-miR-370-3p, WNT7B, 
A

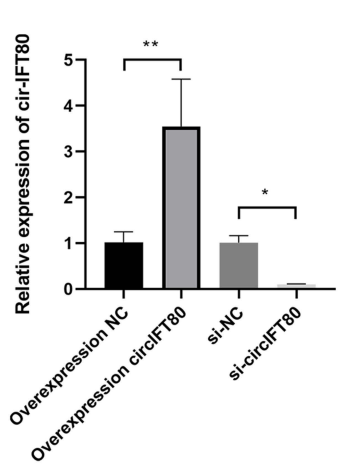

D

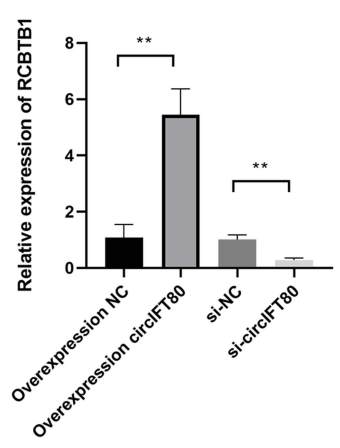

B

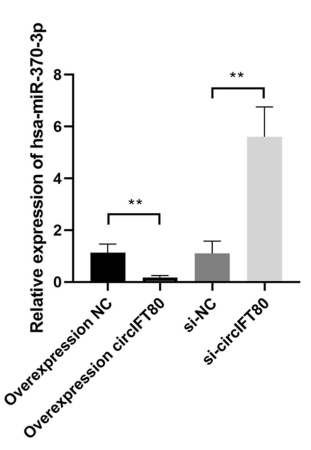

E

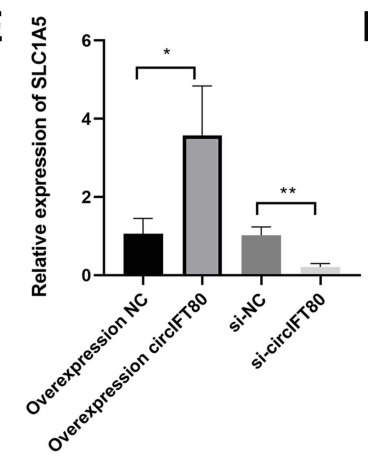

C

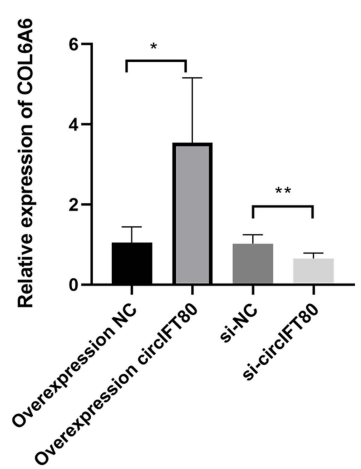

F

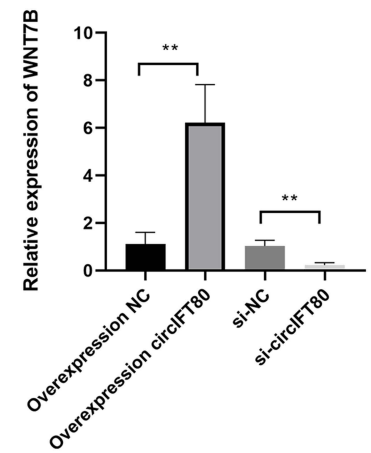

G

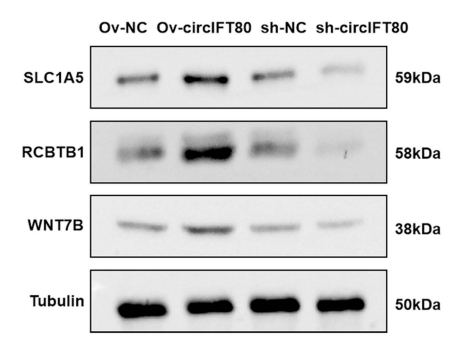

H

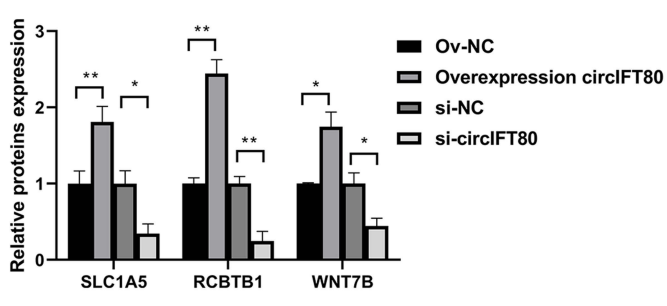

I

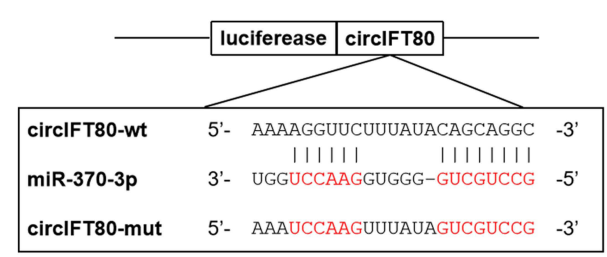

J

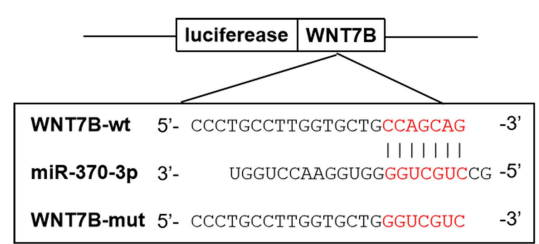

K

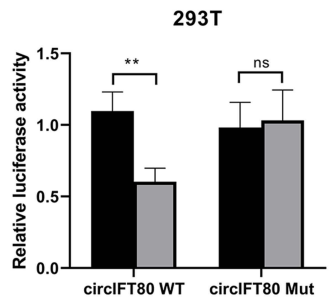

- NC

口 hsa-miR-370-3p
L

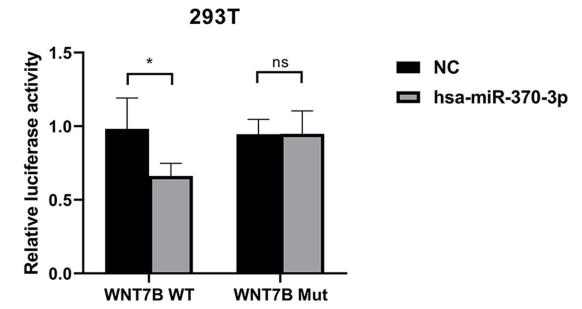

Figure 7 The expression levels of circlFT80, hsa-miR-370-3p, COL6A6, RCBTBI, SLCIA5, and WNT7B in SW480 cells and circlFT80 upregulation of WNT7B expression via sponging hsa-miR-370-3p. (A-F) After overexpressing or knocking out circlFT80 in SW480 cells, qRT-PCR detects the relative expression levels of circlFT80, hsa-miR -370-3p, COL6A6, RCTBBI, SLCIA5, WNT7B in the cells; (G and $\mathbf{H})$ Western blot detects the protein expression levels of RCBTBI, SLCIA5 and WNT7B after overexpression and knockdown with circlFT80; (I and J) The predicted binding sites between hsa-miR-370-3p and circlFT80, hsa-miR-370-3p and WNT7B; (K) The target relationship between hsa-miR-370-3p and circlFT80 was verified by dual-luciferase reporter assay; (L) The target relationship between hsa-miR-370-3p and WNT7B was verified by dual-luciferase reporter assay.** $p<0.01$. ${ }^{*} p<0.05$. vs NC. 
SLC1A5, RCBTB1 and COL6A6 genes on the signal axis are related to cancer.

To confirm the databases-derived data, we analyzed the expression level of circIFT80 in tissues and cells and it could be shown that the expression of circIFT80 is indeed up-regulated. Cell function experiments showed that knockdown of circIFT80 was able to promote apoptosis and inhibit cell proliferation, migration, and invasion. By employing qRT-PCR, we could show that circIFT80 inhibited the expression of hsa-miR-370-3p and promoted the expression of COL6A6, RCBTB1, SLC1A5, and WNT7B in CRC cell lines. The results of the Western blot confirmed that circIFT80 promotes the expression of RCBTB1, SLC1A5, and WNT7B protein. Next, we tried to shed some light on the circIFT80/hsamiR-370/WNT7B interaction network. For this, a dual luciferase reporter assay validated that circIFT80 binds to hsa-miR-370-3p, which in turn targets WNT7B. Taken together, the experimental results indicate that circIFT80 can inhibit the expression of hsa-miR-370-3p, promote the expression of COL6A6, RCBTB1, SLC1A5, and WNT7B, thereby inhibits CRC apoptosis, and promotes CRC proliferation, migration and invasion which is in line with our expectations. Future studies shall verify the interaction of hsa-miR-370-3p and SLC1A5, RCBTB1, and COL6A6 as well as shed light on the signal pathway downstream of the circIFT80/hsa-miR-370-3p/ WNT7B axis in order to provide new ideas for the clinical treatment of CRC.

\section{Conclusions}

In conclusion, we conducted a comprehensive analysis of circIFT80 in CRC tissues and cells, identified miRNAs and mRNAs relevant for CRC development and establishment and constructed a potential regulatory network of circIFT80miRNA-mRNA. The bioinformatics analysis approach represents a useful strategy to explore malignancies from a new perspective. However, future studies are needed to further elucidate the regulatory network between targets and the signaling pathways regulated by their target genes.

\section{Abbreviation}

CRC, Colorectal cancer; circIFT80, hsa _circRNA_0067835; DEMs, differentially expressed miRNA; DEGs, differentially expressed genes; miRNA, microRNA; mRNA, messenger RNA; lncRNA, long noncoding RNA; ncRNA, non-coding RNA; CircInteractome, Circular RNA Interactome; $\mathrm{GO}$, GeneOntology; $\mathrm{CC}$, cell component; $\mathrm{MF}$, molecular function; BP, biological process; KEGG, Kyoto Encyclopedia of Genes and Genomes; PPI, protein-protein interaction; circRNAs, Circular RNAs; ceRNA, endogenous RNA; TCGA, The Cancer Genome Atlas; ATCC, American Type Culture Collection; FBS, fetal bovine serum; DMEM, Dulbecco's modified eagle medium; OD, optical density; CCK-8, Cell Counting Kit-8; qRT-PCR, quantitative RTPCR; Ov, Overexpression; NC, negative control; wt, wildtype; mut, mutant.

\section{Data Sharing Statement}

The datasets generated and analyzed during the current study are not publicly available due to the datasets are related to the patent application but are available from the corresponding author on reasonable request.

\section{Ethics Approval and Consent to Participate}

Ethical approval was provided by the Clinical Ethics Management Committee of Hainan Provincial People's Hospital and written informed consent was obtained from all participants. The number of ethical approval is MedEth-Re [2020] 199. Samples and data were handled according to the declaration of Helsinki. Written informed consent was obtained from each participant.

\section{Acknowledgments}

We are grateful to the Hainan General Hospital and Hainan Affiliated Hospital of Hainan Medical University for their support, and gratefully acknowledge the help provided by the Department of Gastrointestinal Surgery, Hainan General Hospital. We would like to thank the experts of EditSprings (https://www.editsprings.com/) for providing language services.

\section{Author Contributions}

All authors made a significant contribution to the work reported, whether that is in the conception, study design, execution, acquisition of data, analysis and interpretation, or in all these areas; took part in drafting, revising or critically reviewing the article; gave final approval of the version to be published; have agreed on the journal to which the article has been submitted; and agree to be accountable for all aspects of the work. Co-first authors: Ning Liu and Fan Jiang contributed equally to the work and should be regarded as co-first authors. 


\section{Funding}

The work was granted by Hainan Natural Science Foundation Youth Fund [819QN353].

\section{Disclosure}

The authors declare that they have no conflicts of interest in this work.

\section{References}

1. Siegel RL, Miller KD, Jemal A. Cancer statistics, 2020. CA Cancer J Clin. 2020;70(1):7-30. doi:10.3322/caac.21590

2. Bray F, Ferlay J, Soerjomataram I, Siegel RL, Torre LA, Jemal A. Global cancer statistics 2018: GLOBOCAN estimates of incidence and mortality worldwide for 36 cancers in 185 countries. CA Cancer J Clin. 2018;68(6):394-424. doi:10.3322/caac.21492

3. Jathar S, Kumar V, Srivastava J, Tripathi V. Technological developments in lnc RNA biology. Adv Exp Med Biol. 2017;1008:283-323.

4. Yamamura S, Imai-Sumida M, Tanaka Y, Dahiya R. Interaction and cross-talk between non-coding RNAs. Cell Mol Life Sci. 2018;75 (3):467-484. doi:10.1007/s00018-017-2626-6

5. Greene J, Baird AM, Brady L, Lim M, Finn SP. Circular RNAs: biogenesis, function and role in human diseases. Front Mol Biosci. 2017;4:38. doi:10.3389/fmolb.2017.00038

6. Pan H, Li T, Jiang Y, et al. Overexpression of circular RNA ciRS-7 abrogates the tumor suppressive effect of miR-7 on gastric cancer via PTEN/PI3K/AKT signaling pathway. J Cell Biochem. 2018;119 (1):440-446. doi:10.1002/jcb.26201

7. Salmena L, Poliseno L, Tay Y, Kats L, Pandolfi PP. A ceRNA hypothesis: the Rosetta stone of a hidden RNA language? Cell. 2011;146(3):353-358. doi:10.1016/j.cell.2011.07.014

8. Wang P, Sun Y, Yang Y, Chen Y, Liu H. Circ_0067835 knockdown enhances the radiosensitivity of CRC by miR-296-5p/IGF1R Axis. OncoTargets Ther. 2021;14:491-502. doi:10.2147/OTT.S281011

9. Feng W, Gong H, Wang Y, et al. circIFT80 functions as a ceRNA of miR-1236-3p to promote colorectal cancer progression. Mol TherNucl Acids. 2019;18:375-387. doi:10.1016/j.omtn.2019.08.024

10. Ritchie ME, Phipson B, Wu D, et al. limma powers differential expression analyses for RNA-sequencing and microarray studies. Nucleic Acids Res. 2015;43(7):e47. doi:10.1093/nar/gkv007

11. Dudekula DB, Panda AC, Grammatikakis I, De S, Abdelmohsen K, Gorospe M. CircInteractome: a web tool for exploring circular RNAs and their interacting proteins and microRNAs. RNA Biol. 2016;13 (1):34-42. doi:10.1080/15476286.2015.1128065

12. Cava C, Colaprico A, Graudenzi A, Bertoli G, Castiglioni I. SpidermiR: an R/Bioconductor package for integrative network analysis with miRNA data. Int $J$ Mol Sci. 2017;18(2):274. doi:10.3390/ ijms 18020274

13. Yu G, Wang L, Han $\mathrm{Y}, \mathrm{He}$ Q. clusterProfiler: an R package for comparing biological themes among gene clusters. OMICS. 2012;16 (5):284-287. doi:10.1089/omi.2011.0118

OncoTargets and Therapy

\section{Publish your work in this journal}

OncoTargets and Therapy is an international, peer-reviewed, open access journal focusing on the pathological basis of all cancers, potential targets for therapy and treatment protocols employed to improve the management of cancer patients. The journal also focuses on the impact of management programs and new therapeutic

Submit your manuscript here: https://www.dovepress.com/oncotargets-and-therapy-journal
14. Li Y, Li H, Wei X. Long noncoding RNA LINC00261 suppresses prostate cancer tumorigenesis through upregulation of GATA6-mediated DKK3. Cancer Cell Int. 2020;20(1):474. doi:10. 1186/s12935-020-01484-5

15. Bian L, Zhi X, Ma L, et al. Hsa_circRNA_103809 regulated the cell proliferation and migration in $\mathrm{CRC}$ via miR-532-3p/FOXO4 axis. Biochem Biophys Res Commun. 2018;505(2):346-352. doi:10.1016/ j.bbrc.2018.09.073

16. Fang G, Ye B-L, Hu B-R, Ruan X-J, Shi Y-X. CircRNA 100290 promotes CRC progression through miR-516b-induced downregulation of FZD4 expression and Wnt $/ \beta$-catenin signaling. Biochem Biophys Res Commun. 2018;504(1):184-189. doi:10.1016/j. bbrc.2018.08.152

17. Tang X, Sun G, He Q, et al. Circular noncoding RNA circMBOAT2 is a novel tumor marker and regulates proliferation/migration by sponging miR-519d-3p in CRC. Cell Death Dis. 2020;11(8):625. doi:10.1038/s41419-020-02869-0

18. Yuan W, Peng S, Wang J, et al. Identification and characterization of circRNAs as competing endogenous RNAs for miRNA-mRNA in CRC. Peer J. 2019;7(1):e7602. doi:10.77 17/peerj.7602

19. Memczak S, Jens M, Elefsinioti A, et al. Circular RNAs are a large class of animal RNAs with regulatory potency. Nature. 2013;495 (7441):333-338. doi:10.1038/nature11928

20. Hansen TB, Jensen TI, Clausen BH, et al. Natural RNA circles function as efficient microRNA sponges. Nature. 2013;495 (7441):384-388. doi:10.1038/nature11993

21. Lin L, Wang D, Qu S, Zhao H, Lin Y. miR-370-3p alleviates ulcerative colitis-related colorectal cancer in mice through inhibiting the inflammatory response and epithelial-mesenchymal transition. Drug Des Dev Ther. 2020;14:1127-1141. doi:10.2147/ DDDT.S238124

22. Jiang YJ, Wei TR, Li W, Zhang RH, Chen MG. Circular RNA hsa circ 0002024 suppresses cell proliferation, migration, and invasion in bladder cancer by sponging miR-197-3p. Am J Transl Res. 2019;11(3):1644-1652.

23. Jiang S, Li Q, Liu Y, Zhang H, Zhang P. Activation of WNT7b autocrine eases metastasis of CRC via epithelial to mesenchymal transition and predicts poor prognosis. BMC Cancer. 2021;21 (1):180. doi:10.1186/s12885-021-07898-2

24. Huang F, Zhao Y, Zhao J, Wu S, Tao Z. Upregulated SLC1A5 promotes cell growth and survival in CRC. Int J Clin Exp Pathol. 2014;7(9):6006-6014.

25. Mauduit O, Brulard C, Lesluyes T, et al. RCBTB1 deletion is associated with metastatic outcome and contributes to docetaxel resistance in nontranslocation- related pleomorphic sarcomas. Cancers. 2019;11(1):81. doi:10.3390/cancers11010081

26. Long R, Liu Z, Li J, Yu H. COL6A6 interacted with P4HA3 to suppress the growth and metastasis of pituitary adenoma via blocking PI3K-Akt pathway. Aging. 2019;11(20):8845-8888. doi:10.18632/ aging. 102300

agents and protocols on patient perspectives such as quality of life, adherence and satisfaction. The manuscript management system is completely online and includes a very quick and fair peer-review system, which is all easy to use. Visit http://www.dovepress.com/ testimonials.php to read real quotes from published authors. 